\title{
LA REPÚBLICA, EL ESTADO Y EL MERCADO EN EDUCACIÓN
}

\author{
Carlos Ruiz Schneider \\ Universidad de Chile \\ cruizsch@gmail.com
}

\begin{abstract}
Resumen
En este artículo se comparan dos teorías normativas sobre la educación en Chile, durante los siglos XIX y XX, la teoría que sustenta la educación en la idea de república, fundamentalmente en el siglo XIX, y el modelo que impone el régimen de Pinochet que recurre a la utilidad y la libertad de mercado como conceptos justificatorios.

La teoría republicana argumenta a favor de un papel central del Estado en el diseño de un sistema educacional público, universal, obligatorio (por lo menos hasta el nivel secundario) y gratuito, que se entiende como fundamental para la formación común del ciudadano y para la construcción de una sociedad de iguales. Se puede decir que en esta perspectiva, la educación es una propiedad común, un bien público del que nadie, en principio puede ser excluído.

Para el modelo de mercado, la educación es un bien de consumo -o en otras perspectivas, una inversión-que significa altas tasas de retorno para los individuos y que por esta razón debe ser pagada por los usuarios. Desde otra mirada, un sistema privado de educación se justifica también en términos de la libertad de los agentes económicos para ofrecer bienes educacionales en el mercado y la libertad de escoger de consumidores potenciales. En el artículo se argumenta que, por lo menos históricamente, en Chile, un modelo similar al republicano existió hasta 1973, el que coexistió con un sistema educacional privado. En el debate actual, esta coexistencia entre un sistema público y uno privado se considera positiva. En oposición a esto, el actual modelo educacional, fundamentalmente de mercado, es excluyente, incapaz de coexistir con un sistema público fuerte y de calidad, y tiende por lo tanto a la desestatización progresiva de toda la educación.
\end{abstract}

Palabras Clave: república, autogobierno, igualdad, liberalismo, Estado, mercado, utilidad, libertad.

\section{Abstract}

This paper compares two normative theories of education in Chile, one centered in the republican tradition, mainly in the XIX and partly in the XXth century, and the other imposed by the military dictatorship and who uses the market freedom and the market efficiency as justificatory devices.

The republican theory argues in favor of a central role of the state in the design of a public, universal, obligatory and tuitionless educational system, understood as essential to the formation of citizens in common and to the construction of an egalitarian society. One can think of education, in this perspective, as a common property, a public good of which nobody can, in principle be excluded. 
To the market theory, education is conceived as a consumer good -or, in another version, as an investment-who means high rates of return to individual users and for that reason must be paid by them. From another perspective, a private system of education is justified in terms of the freedom to offer educational goods in the market, and the freedom to choose this goods by theconsumers.

In Chile an educational system very similar to the republican one, has existed till 1973. This system has had a relatively peaceful coexistence with private education in all levels. In current discussions on education, this mixed system is considered a good thing. The paper argues that this coexistence is no more possible with the actual market model who seeks to exclude any other system, which is incompatible with a strong public system of high quality and who points therefore to the destatisation of all education.

KEY WORDS: Republic, self-government, equality, liberalism, state, market, utility, freedom.

$\widehat{R A}$

Mi interés por las relaciones entre república, Estado y mercado en educación surge de los debates políticos recientes, pero de gran importancia en Chile, por lo menos a partir del año 2006, y que tienen su mayor expresión en el año 2011 a partir del desarrollo del gran movimiento social que tuvo como protagonistas a los estudiantes secundarios, los estudiantes universitarios y a organizaciones de maestros, que exigían el fin del sistema educacional vigente. Este movimiento, expresión, tal vez, de lo que podría llamarse una insurgencia democrática, congregó en las calles de Santiago -pero también fue muy fuerte en todas las provincias- a más de 1.000 .000 de estudiantes, con la mayoría de los establecimientos secundarios tomados, en base a unas demandas y a un estilo de movilización muy novedoso en la escena política chilena. En efecto, la organización de este movimiento ha sido muy horizontal, con escasas relaciones con los partidos políticos, aunque apoyado por organizaciones políticas estudiantiles y luego masivamente por sectores medios y populares.

Este retorno de la educación al debate público en Chile me animó personalmente a darle forma de libro a una serie de ensayos sobre educación y universidad escritos a lo largo de varios años, lo que derivó en un texto titulado De la República al mercado. Ideas educacionales y politica en Chile (Santiago, LOM, 2010) y es en base a este libro y al movimiento del año 2011 que querría hacer algunas reflexiones hoy sobre las relaciones entre la república, el Estado y mercado en educación.

Parece claro, en una primera aproximación, que la idea de república, el Estado y el mercado son conceptos que juegan un papel importante a la hora de caracterizar los principios políticos que organizan los sistemas educacionales modernos.

El Estado tiene un lugar preeminente en muchas teorías normativas de la educación. El concepto del mercado ocupa ese lugar en realidad solo en las teorías que se inspiran en el neo-liberalismo, pero estas teorías tienen hoy mucha importancia.

Hay que advertir de entrada que, sin embargo, estos conceptos tan generales no agotan el campo de los posibles. Por ejemplo, las visiones anarquistas en educación ciertamente no le otorgan al Estado ningún papel protagónico en la autoeducación del 
pueblo, salvo en un sentido negativo. Y habría que decir que, salvo en sus versiones actuales de extrema derecha, como el anarco-capitalismo, tampoco le otorgan ese papel al mercado. Por otra parte, hay también en muchos casos, modelos mixtos, que recurren al Estado y al mercado como conceptos reguladores.

Como quiero centrar mi reflexión en Chile, en los siglos XIX y XX, descartaré las visiones anarquistas o próximas al anarquismo, que son importantes, pero que no han sido muy estudiadas. Por otra parte, el republicanismo del siglo XIX y comienzos del siglo XX tiene varios puntos de cercanía con las concepciones socialistas que naturalmente, también subrayan con fuerza el rol del Estado en educación. Dejaré para otra el análisis de las ideas socialistas en educación, en parte porque es para mí una tarea pendiente y en parte porque el republicanismo experimenta desde los años 1970 una importante revitalización, una de cuyas aristas es una crítica actualizada del neo-liberalismo, que analizaremos en el siglo XX.

Por otras razones, dejaré para el final las concepciones o modelos que intentan articular Estado y mercado, que me parecen estar en graves dificultades hoy, precisamente por el papel fundamental que se le asigna hoy al mercado en educación y por el tipo de teoría en el que se basa este papel.

Las concepciones sobre un rol preeminente del Estado en la educación tienen sus raíces en el supuesto de que la forma de Estado más defendible o justificable en la modernidad es la república y una república democrática.

Si el principio de la república es la virtud política, es decir, la preeminencia constante del interés general por sobre el interés privado, o del bien público sobre el privado, esto quiere decir que la educación es fundamental porque esa preeminencia -como lo sostiene, por ejemplo, Montesquieu- no es natural, y todo depende en la república democrática de una ciudadanía activa y comprometida con la defensa de este interés general. Una tarea central de la república es, pues, la de formar al ciudadano y esa tarea es la tarea de la educación.

Como lo ha mostrado Quentin Skinner (1997) en un notable ensayo, la tradición republicana no le asigna necesariamente este papel protagónico al Estado, aunque sí lo hacen dos de sus variantes: el modelo republicano revolucionario francés de fines del siglo XVIII y luego también el de fines del siglo XIX, que será, por ejemplo, de gran importancia para el desarrollo de la reflexión de los teóricos educacionales chilenos como, por ejemplo, Valentín Letelier uno de los grandes defensores de la idea de educación pública estatal.

¿Cuál es el fundamento de esta relación fuerte entre república y Estado en el republicanismo francés del siglo XIX? 
Disponemos para examinar esta relación de varios trabajos. Dos de los más importantes son los libros de Claude Nicolet, L'idée republicaine en France, y el de Jean-Fabien Spitz, Le moment republicain en France. A esto me parece que habría que agregar una ponencia del profesor Spitz del año 2008, en Chile, publicada en el libro de Marcos García de la Huerta y Carlos Ruiz, República, liberalismo y democracia, titulado "El republicanismo francés".

La tradición republicana francesa es muy distinta de la estadounidense, que ha sido más estudiada por los autores republicanos contemporáneos. La marca, naturalmente, la Revolución de 1789, cuyo radicalismo, en especial en el período de la Convención, había enfriado el entusiasmo original de los líderes de la emancipación americana con la República. El proceso revolucionario francés es, no obstante, de una gran riqueza, la que va desde el radicalismo democrático de los jacobinos, hasta el republicanismo liberal y burgués del Directorio. Pero ni siquiera el Imperio napoleónico se desvincula del legado social revolucionario, como lo muestra la elaboración del Código Civil, que desvincula la propiedad de las clases aristocráticas y continúa el desarrollo de la secularización de una serie de instituciones, como la familia.

De acuerdo al historiador Claude Nicolet, las conquistas irreversibles que los republicanos franceses identifican con la Revolución, a pesar de las vicisitudes que experimentan estos logros durante el siglo XIX, son, en primer lugar, "la igualdad jurídica de los ciudadanos y la desaparición o cuasi-desaparición de todo privilegio en este dominio... En este orden de ideas, el Código Civil aparece como la suma del legado revolucionario".

Según Nicolet, "la segunda gran adquisición es la idea de la soberanía nacional... De (ella)... deriva la idea de unidad nacional, en nombre de la cual fue transformada, desde 1791, la vieja organización provincial más o menos particularista”.

Por último, sostiene este autor, los republicanos defienden como indispensable la representación política, aunque se abran, sobre todo en 1848 , en una cierta medida hacia algunas formas de democracia directa.

En Le moment republicain en France, que se refiere fundamentalmente al fin del siglo XIX y comienzos del siglo XX, Jean-Fabien Spitz subraya en el republicanismo francés también otras tendencias, en especial, una cierta idea de libertad como nodominación, en el sentido que tiene la libertad republicana para Philip Pettit, lo que lo lleva a rechazar la idea liberal de libertad negativa, en base al argumento que las interferencias que no son arbitrarias en la vida de los individuos, son legítimas. Interferencias no arbitrarias son, por ejemplo, las que derivan de las leyes, que no necesariamente disminuyen, sino que pueden ensanchar nuestras libertades, lo que justifica plenamente para el republicanismo francés la intervención del Estado y la regulación de la vida social por el derecho. Este argumento tiene, naturalmente, una gran importancia para los debates educacionales, ya que de este modo, la intervención del Estado en la educación puede ser vista como liberadora en base a muchas de sus características, como, por ejemplo, la de hacer posible en base a una ley, como la de 1881 en Francia, o la de 1879 en Chile, un sistema educacional gratuito en todos 
sus niveles, lo que libera, en parte, a los estudiantes de la presión de las familias, las estrategias de los filántropos o la Iglesia ${ }^{1}$.

Desde otras perspectivas, Michael Sandel subraya en el republicanismo la importancia de la idea de autogobierno, lo que también tiene gran relevancia en este estudio porque, si agregamos este rasgo a la idea de libertad como no-dominación y la crítica de la libertad negativa, podemos encontrar aquí otra razón para justificar la intervención del Estado en la educación, esta vez en base al objetivo de la formación de los ciudadanos que requiere una república democrática.

Para los autores republicanos franceses de fin de siglo, como Léon Bourgeois, Celestin Bouglé o Emile Durkheim, la intervención del Estado es esencial para que pueda desarrollarse una sociedad con igualdad de oportunidades, que el mercado es incapaz de establecer y que es, sin embargo, una condición necesaria de la convivencia política legítima en la modernidad, donde las únicas desigualdades tolerables son las que se basan en el mérito. Aunque este nuevo republicanismo francés será importante en Chile, sobre todo a comienzos del siglo XX, algunas de sus características pueden percibirse en el pensamiento de autores de fines del siglo XIX, como Valentín Letelier ${ }^{2}$.

En síntesis, tres son las ideas centrales del republicanismo francés, según lo sostendrá Spitz en una ponencia el año 2008. En primer lugar, la idea de que la "igual ciudadanía, la libertad y la democracia son incompatibles con la dependencia económica”. En segundo lugar, la idea de que las "relaciones de dependencia económica no se resuelven en el mercado(...):. La evolución espontánea de las relaciones de competencia no conduce a la generalización de la independencia sino, al contrario, a la concentración de los medios de trabajo y a la destrucción de las bases para la independencia para la más grande mayoría". Y tercero, la idea de que solo la repartición más justa de "los medios de la independencia, sólo esta construcción de una estructura social que se esfuerce en garantizar a cada uno un acceso a los medios para desarrollar sus facultades y para vivir de manera autónoma, engendra obligación y legitimidad".

En el caso chileno, se pueden encontrar principios republicanos operando en la institucionalización de la educación a lo largo de todo el siglo XIX ${ }^{3}$. Pero la relación entre el republicanismo y el Estado es especialmente clara en la obra de Valentín

1 Según la historiadora Sophie Wahnich, este es precisamente el argumento en que se apoya la defensa de la gratuidad del sistema educacional francés a partir de fines del siglo XIX. Ver, sobre este punto, Wahnich, Sophie, "Defender la gratuidad escolar hoy (como ayer)", en C. Gutiérrez, A. Martin, C. Ruiz y P. Vermeren (2011).

2 Véase sobre estos análisis el libro antes citado de Jean-Fabien Spitz, Le moment républicain en France. En este libro, Spitz sostiene que el proyecto republicano francés es "profundamente individualista" y se centra en la "igualdad de oportunidades" como la única condición que puede "legitimar la búsqueda individual del bienestar", y que, sin embargo, ésta solo puede ser garantizada por la intervención del "poder público en el juego social".

3 Se puede consultar sobre este punto mi libro De la República al mercado. Ideas educacionales y politica en Chile (2010). 
Letelier. Esta temática aparece, en especial, en el debate que lo opone a los partidarios del individualismo liberal de Adam Smith, Humboldt, John Stuart Mill y Herbert Spencer. En su oposición a este liberalismo individualista, Letelier reitera, en primer lugar, como positivista militante que es, la homogeneidad necesaria a la instrucción general como condición de cohesión social. Esta condición básica no podría ser lograda por la educación privada y su anárquica oferta de visiones de mundo, sino que debe lograrse por la educación pública del Estado.

Pero en segundo lugar, Letelier defiende también a la educación pública en función del valor republicano de la igualdad. Desde un punto de vista político, dice Letelier, "las democracias tienen que dar la preferencia a la educación pública porque la escuela común es una institución esencialmente democratizadora... Según lo han demostrado muchos educacionistas, forma la escuela una como república sujeta al régimen de la igualdad, república en que desaparecen las distinciones sociales de la fortuna i la sangre para no dejar subsistentes más que las de la virtud i el talento"4. A lo que agrega que "donde deja de ser pública, la enseñanza pierde su carácter democrático e igualitario, se convierte en simple adorno de las clases oligárquicas, i por el mismo hecho, se inhabilita para cumplir sus fines peculiares" 5 .

Frente a los partidarios de esta especie de laissez faire libertario, sostiene Letelier que la educación no es un bien de consumo: ni los profesores son productores de una industria, ni los estudiantes son consumidores. En el sentir de muchos autores de nuestros días, afirma, y su discurso suena muy actual, "esta industria debe ser tan libre que un ganapán cualquiera no tenga más trabas para establecer una escuela que para abrir una tienda. Nadie puede suponer que el público carezca de competencia para juzgar entre enseñanza i enseñanza, cuando la tiene para juzgar entre mercadería i mercadería". A lo que Letelier responde que el argumento reposa sobre una falsa analogía, porque a diferencia del caso del consumo, "el que no tiene instrucción alguna carece de competencia para elegir entre enseñanza i enseñanza", como se supone la tiene un consumidor cualquiera para elegir entre mercadería y mercadería (Y aún así, diríamos hoy, es cada vez más necesaria la información y la educación del consumidor). Una industria se establece, nos dice Letelier, cuando la reclama el consumo y el consumo la reclama en función de necesidades; lo contrario ocurre con la enseñanza: "cuanto mayor es la ignorancia, tanto más se necesita la instrucción i tanto menos generalmente se siente su necesidad. Los economistas sostienen que a virtud de la lei de la oferta i la demanda, cuando el estado no interviene, la iniciativa particular mejora la enseñanza i funda escuelas dondequiera se las necesita. Pero en el hecho ocurre una cosa diferente: las escuelas se abren en las más grandes poblaciones, donde es mayor la cultura i menor la necesidad, i no en las poblaciones más atrasadas, donde es mayor la necesidad, por 
ser menor la cultura. No son estas, entonces, empresas industriales, sujetas a la lei de la oferta i el pedido. Son empresas morales, sujetas a las necesidades de la cultura"7.

Hay por último, para Letelier, una necesidad estrictamente política, el autogobierno, que requiere de la instrucción pública y sus características, la universalidad, la gratuidad y la obligación. Un pueblo ignorante, nos dice Letelier, "puede ser gobernado...pero sólo un pueblo ilustrado puede gobernarse...Si, pués, es dable prescindir de la instrucción en los estados autocráticos, se la debe generalizar mucho antes de generalizar el sufragio, en aquellos que tienen tendencias democráticas"8.

Como se ve en estos últimos textos, es, sobre todo en la defensa de lo público y de la educación pública como forma de sociabilidad y preparación igualitaria para el autogobierno, que nos encontramos en Letelier con una problemática republicana.

Lo que parece actual e importante en Letelier y la tradición republicana en la educación que hemos descrito consiste, sobre todo, a mi juicio, en su énfasis en que la educación y el saber no son mercaderías, ni responden a la lógica de las preferencias, sino más bien a la de las necesidades sociales que requieren provisión pública. La comprensión de sí mismo, del mundo y del otro, que constituyen el núcleo de lo que un sistema educacional debiera promover en los estudiantes, no se busca en función de la venta o la ganancia, la competencia o la demanda.

Muchas veces, sin embargo, el mismo Letelier se refiere a sus principios como principios liberales. ¿Por qué insistir entonces en que su concepción de la educación es republicana? ¿Cuál es, en definitiva, la relación entre Letelier y el liberalismo?

Respondiendo esta pregunta nos aproximamos más al centro mismo de las ideas políticas de Letelier, importantes para apreciar mejor su combate pedagógico.

En un primer sentido, en un sentido muy amplio, Letelier se identifica, sin duda, con el liberalismo. Es porque entiende al liberalismo en este sentido amplio, como coextensivo de la modernidad, de la libertad de conciencia, de expresión, etc.

Explicándonos el sentido global de su libro La lucha por la cultura, nos dice, por ejemplo, que todas sus piezas "están dirijidas a un solo propósito: el de fomentar i defender la cultura liberal de la República contra las artes de una reacción más peligrosa por su audacia que por su fuerza" (Letelier 1895: VI). Ahora bien, continúa el análisis de Letelier, el liberalismo "que se ha difundido como doctrina hasta imponerse a sus adversarios, se ha debilitado como fuerza política. Antes, cuando era menos grande, era más fuerte porque tenía más cohesión i gozaba de mayor popularidad. Hoy, cuando todo Chile es liberal, este partido no tiene pueblo porque, haciendo profesión de libre cambio, se muestra insensible a las necesidades populares; ni tiene tampoco la fuerza que le corresponde porque, haciendo profesión de independencia, siembra entre sus 
prosélitos el espíritu de indisciplina. De cierto esta no es una época de decadencia liberal; pero es una época de anarquía liberal" (Letelier 1895: VI, VII).

Como puede vislumbrase en estas páginas, Letelier es hostil, en cambio, a un segundo sentido de liberalismo que identifica con el libre cambio. A su triunfo en el interior del liberalismo, lo vincula Letelier con el peligro de que tomen nuevas fuerzas tanto la reacción conservadora como la anarquía revolucionaria.

Para entender con más hondura esta posición de Letelier puede ser conveniente analizar brevemente su concepción sobre los fines de la política. Nos dice en el ensayo "Ellos i nosostros. O sea los liberales i los autoritarios", que "el fin de la política no es realizar tal o cual principio ideal sino satisfacer las necesidades sociales. Las doctrinas se forman para los pueblos, no los pueblos para las doctrinas"(Letelier 1895, p. 23). Y en este sentido, Letelier reitera al terminar el ensayo que "el fin de la política no es la libertad, no es la autoridad, ni es principio alguno de carácter abstracto, sino que es el de satisfacer las necesidades sociales para procurar el perfeccionamiento del hombre i el desarrollo de la sociedad" (Letelier 1895, p. 31).

Piensa Letelier que es por esta razón por la que "debemos juzgar anticientífica aquella escuela que en nuestros tiempos enseña ser la libertad personal, la de pensamiento, la de trabajo i la igualdad, derechos imprescriptibles, inalienables e inherentes a la naturaleza humana (...) porque las libertades todas son simples medios de desarrollar las fuerzas sociales (...)” (Letelier 1895, p. 24).

Letelier ahonda todavía esta crítica, de cuño claramente positivista, de la noción metafísica de libertad, que le parece estar en el origen de la decadencia del liberalismo en el primer sentido, en palabras como las siguientes: "debemos estirpar de nuestro espíritu la preocupación metafísica i revolucionaria que supone ser la libertad una panacea propia a curar todas las enfermedades del organismo social en todos los grados de su desarrollo" (Letelier 1895, p. 22). Y prosigue algo más adelante diciéndonos que nada ha colaborado más, en nuestro tiempo, al avasallamiento de algunos pueblos "que la absoluta confianza de los liberales en el régimen de la libertad. Convencidos (...) de que ella tiene ciertas virtudes ocultas para curar todos los males, han solido renunciar a toda cooperación de la parte del Estado; e imaginándola dotada de una vida propia, inmortal e independiente de las condiciones sociales han solido anular los poderes llamados a protejerla i han dejado imprudentemente desarrollarse fuerzas reaccionarias. En tamaños errores está el mayor peligro del liberalismo"(Letelier 1895, pp. 22-23).

En este sentido Letelier aprueba el pensamiento de Bentham, que "llamó sofismas políticos esos principios aprióricos que cada partido aprueba sin prueba alguna a manera de pauta para resolver todos los problemas de gobierno" (Letelier 1895, p. 23).

Con el autor utilitarista -y también con Auguste Comte- Letelier piensa que se debe eliminar de la política la noción metafísica y anárquica de los principios absolutos.

En su conocido artículo sobre "Los pobres", publicado en el diario La Ley, Letelier prosigue con su análisis de los problemas del liberalismo, ahora poniendo énfasis en el librecambismo. 
Si la metafísica anárquica de la libertad es lo que le deja libre el camino a la reacción conservadora - dice- el librecambismo lo que hace es dejar libre la vía a la revolución socialista.

Para explicar mejor este tema, sostiene Letelier que : "Es evidente, por ejemplo, que la libertad de contratar tiene en nuestros códigos un alcance mucho mayor que en los tiempos de Justiniano. La disolución de las corporaciones industriales, la abolición de la servidumbre i la abrogación del sistema de privilegios mercantiles, han hecho a cada uno árbitro de su persona, de su trabajo i de sus obras. Pero esta nueva situación que ha atizado la lucha por la vida, ha hecho a los desvalidos víctimas de los fuertes i de los poderosos. El régimen de libertad, que es un régimen esencialmente negativo, que no es régimen de garantía, es el mejor de los estados jurídicos para los que contratan i obran en condiciones de relativa igualdad. Mas cuando no existe esta igualdad, la libertad es una irrisión para los débiles, porque "no hai desigualdad mayor que la de aplicar un mismo derecho a los que de hecho son desiguales".

Después de estas denuncias de la libertad metafísica del liberalismo y de lo irrisorio de la libertad negativa en la práctica, aborda Letelier el carácter del Estado liberal. Su denuncia es también categórica. Dice de partida que los burgueses terminaron por construir un Estado burgués:

"Desde el día en que (la burguesía) acometió la grande empresa de la reorganización del Estado antiguo, inventó doctrinas que enseña en sus cátedras i que difunde por medio de sus diarios, dirijidas a justificar una política negativa i egoísta que dá a los burgueses todo lo que les conviene i niega a los proletarios todo lo que necesitan. Aludo a las doctrinas del libre cambio i el individualismo. En efecto ¿qué es lo que necesitan los grandes para explotar a los pequeños?...libertad i nada más que libertad, o sea la garantía de que el Estado no intervendrá en la lucha por la existencia para alterar el resultado final a favor de los desvalidos. Eso es lo que el libre cambio da a los burgueses...

I qué es lo que necesitan los desvalidos...Sólo protección i nada más que protección o sea la garantía de que el Estado igualará las condiciones de los combatientes, dando armas a los débiles para luchar con los fuertes. Esto es lo que el individualismo niega a los desvalidos"

Después de este diagnóstico del significado social del liberalismo, Letelier esboza las consecuencias políticas de su implementación en la práctica. "Todo -dice- lo que el liberalismo de nuestros días ha hecho por los pobres se reduce sustancialmente a la instrucción i al sufragio; esto es, a ilustrarle para que conozca mejor sus miserias i a armarle para que pueda exigir por si mismo el remedio de sus males. Sorprenderse del aparecimiento del socialismo es sorprenderse de que la instrucción popular de su fruto más genuino, el de dar capacidad al pueblo para estudiar sus propias necesidades".

Letelier piensa que solo el abandono en que se ha dejado a los intereses populares, consecuencia del liberalismo, puede explicar una anomalía que considera en extremo peligrosa, esto es, "que en el seno de nuestras sociedades igualitarias se estén renovando las luchas de clases, fatales para el funcionamiento regular de la verdadera democracia" (Letelier 1896). 
Este es el marco político mucho más amplio en el que Letelier piensa que tiene que actuar el radicalismo, haciendo suya la causa de los pobres. Este es también el marco histórico que da sentido a su proyecto educacional y su orientación hacia la producción de cohesión social, bajo la dirección de una nueva clase dirigente, producto de la universidad y la cultura y no del nacimiento o la riqueza. Es por esto también que preferimos caracterizar su concepción política y educacional como republicana y no simplemente como liberal. La problemática de Letelier no se reduce a exigir pluralismo y tolerancia religiosa -aunque persigue esto también-, ni apunta a organizar una cruzada contra la intervención del Estado en la educación, sino que, mucho más cercana a una problemática republicana del autogobierno y la libertad como no-dominación, implica claramente una intervención estatal que desde los bancos de la escuela logre contribuir a la formación de los ciudadanos en Chile, lo que no se logra sino en base a una escuela común, en la que los hijos de los ricos, junto a los de los pobres aprendan a considerarse como los iguales que son, junto con el aprendizaje de los rudimentos del autogobierno.

Aparte el republicanismo, y a partir de mediados del siglo XIX, la doctrina socialista, en especial en su vertiente social-demócrata, es la principal defensora de un papel central del Estado en la educación. Los fundamentos de esta intervención fuerte del Estado coinciden, en parte, con las ideas que destaca Spitz para el republicanismo francés de fines del siglo XIX y comienzos del XX. En Chile, las ideas socialistas próximas a la social-democracia serán también muy influyentes en educación con los gobiernos radicales, entre 1938 y 1952. El socialismo de raíz marxista comparte en gran medida estos enfoques, pero la teoría marxista es mucho más crítica frente a las posibilidades de cambio del Estado capitalista. La mayor parte de los enfoques marxistas sobre la educación se centran por esto en el papel de las instituciones educativas en la construcción de una hegemonía social. La obra de Antonio Gramsci es aquí paradigmática, aunque desde fines de los 1960 hay autores como Paulo Freire que subrayan el papel liberador que puede jugar una educación popular renovada.

Sin duda una muy buena ilustración de cómo un sistema educacional puede ser organizado en el marco del funcionamiento de un mercado capitalista es el caso de Chile desde fines de los 1970, bajo la dictadura militar. Como parece también bastante claro, lo fundamental de este modelo educativo continúa hasta hoy, sin grandes modificaciones estructurales, aunque hay un importante aumento del gasto, fundamentalmente privado, en educación durante los gobiernos de la Concertación por la Democracia.

En una carta que Pinochet dirige al Ministro de Educación Gonzalo Vial y que acompaña a la Directiva Presidencial sobre Educación Nacional de marzo de 1979, leemos por ejemplo que, en adelante, el Estado “centrará el énfasis en la educación básica $\mathrm{y}$, a cualquier costo, cumplirá su deber histórico y legal de que todos los chilenos, no sólo tengan acceso a ella, sino que efectivamente la adquieran y así queden capacitados para ser buenos trabajadores, buenos ciudadanos y buenos patriotas" (El Mercurio, 5 
de marzo, 1979). Esta primera afirmación fundamental, que se repite en la Directiva, significa, en definitiva, el anuncio de que el Estado deja de guiarse por los principios republicanos, que caracterizan al sistema educacional durante prácticamente toda la historia independiente de Chile.

En efecto, que el Estado enfatice en educación solo el tramo básico significa, por una parte, que su acción está dirigida a la formación de un mínimo funcional al desempeño productivo, a lo que se agrega una vaga alusión de contenido nacionalista. En este sentido, continúa la carta, "(alcanzar) la educación media, y en especial, la superior, constituye una situación de excepción para la juventud, y quienes disfruten de ella deben ganarla con esfuerzo (...) y además debe pagarse o devolverse a la comunidad nacional por quien pueda hacerlo ahora o en el futuro (...) “ (ibíd.) Como vemos, el texto de la carta, que resume en general la Directiva, es claro en establecer este retiro del Estado de su función fundamental de apoyo a todo un recorrido educacional que no se limita al tramo básico; en realidad, la propuesta de Pinochet significa una ruptura completa con toda forma de contribución de la educación a la formación de la ciudadanía, lo que ha sido tradicionalmente, en Chile, una función de la educación secundaria. En esta nueva perspectiva, entonces, la educación aparece vista como un bien de consumo, por el que los usuarios deben pagar. El perfil de los estudiantes se modifica fundamentalmente, y pasa del ciudadano al consumidor ${ }^{9}$.

Más en general, el texto implica también una ruptura con el principio de igualdad de oportunidades, cuyo rango de aplicación no se agota, naturalmente, en la enseñanza básica. Este abandono del principio de igualdad de oportunidades educativas, y del compromiso estatal en apoyarla, significa en realidad un refuerzo a la selección fundada en criterios económicos y, por lo tanto, a la desigualdad social. En este sentido, esta carta muestra el carácter radicalmente reaccionario de la política educativa de la dictadura militar, cercana a los autores conservadores que en el siglo XIX se oponen a la expansión de la educación pública y que marcha exactamente en sentido inverso a las tendencias principales de la evolución de las políticas educacionales en el mundo,

9 En un ensayo fundador del modelo educacional chileno, el economista Milton Friedman, del Departamento de Economía de la Universidad de Chicago, había sostenido que "En la educación, los padres y los hijos son los consumidores y el profesor y el administrador de la escuela, los productores. La centralización educativa ha supuesto... una reducción de las posibilidades de elección del consumidor y un incremento del poder de los productores ... (cuyos)... intereses se pueden satisfacer con una mayor centralización y burocratización, aunque estas no satisfagan los de los padres" (p. 220). En un pasaje sobre educación superior del mismo ensayo leemos: "En las instituciones privadas (de educación superior) la situación es muy distinta... Lo importante es que los estudiantes son los principales clientes; pagan por lo que se les da y quieren recibir el equivalente a su dinero... La universidad vende enseñanza y los estudiantes la compran. Como en la mayoría de los mercados privados, ambas partes tienen fuertes incentivos que ofrecerse mutuamente" (p. 244). Véase Milton Friedman, “¿Qué falla en nuestras escuelas?, en Milton y Rose Friedman, Libertad de elegir. Hacia un nuevo liberalismo económico. Recuérdese que ya a fines del siglo XIX, Valentín Letelier había detectado una falacia de falsa analogía en este tipo de argumento. 
precisamente en los años 1970. En general, por ejemplo, en este momento, en los países avanzados, la educación secundaria y también la superior no se ven ya como privilegios, como en el siglo XIX, sino como un derecho que tienen los jóvenes, no solo en virtud de su formación como ciudadanos, sino también como un piso mínimo para cualquier desempeño laboral y como un elemento central en lo que algunos están identificando ya como la "sociedad del conocimiento".

Al analizar estos cambios radicales en educación, no debemos olvidar que el nuevo modelo que se busca implementar no es fruto de la deliberación sino de decretos leyes de un régimen autoritario. Un sistema de mercado en educación no tiene necesariamente que surgir del fiat de una autoridad dictatorial o tiránica, pero de hecho así surge la nueva institucionalidad educacional en Chile, institucionalidad que es reafirmada por otro fiat de la autoridad dictatorial, la Ley Orgánica Constitucional de Educación, dictada en 1990 como un muro prácticamente infranqueable que asegura la continuidad del modelo bajo los gobiernos democráticos.

En segundo lugar hay que decir que, como queda de manifiesto en el discurso de la Directiva, la educación se concibe ahora como un bien fundamentalmente privado. Los agentes fundamentales de la educación son los individuos y sus familias que defienden su libertad negativa individual de no ser interferidos por agencias del Estado o que están empeñadas en una estrategia y un cálculo de utilidad que parte de la base de que los retornos económicos de la educación son altos y que benefician a los individuos, lo que descarta la necesidad o la obligación de una intervención del Estado en su provisión.

Estas dos líneas de defensa del mercado se han dado en Chile.

Una defensa cercana al utilitarismo la encontramos en uno de los ensayos más extremos que apoyan en Chile el modelo de mercado educativo, el artículo titulado "El sistema de subvenciones en educación. La experiencia chilena", escrita por el actual director de CODELCO, Gerardo Jofré y publicada en Estudios Públicos N 32 de 1988 y reeditado en el libro Educación chilena. Doctrina y políticas, editado por Tomás Mc Hale en la Editorial Universitaria en 1989 y que recoge los textos esenciales sobre educación durante el período dictatorial.

Desde la perspectiva de Jofré, dado el alto nivel de utilidad de los servicios educacionales y su recuperación por los individuos, en estricto rigor no se justifica la intervención del Estado en la educación. Es más, la intervención estatal es peligrosa para la sociedad ya que "tiene costos significativos para la economía que el Estado entregue subsidios. Estas transferencias no son neutras...desincentivan al esfuerzo y al trabajo a los receptores de los subsidios, quienes tendrán acceso a un mayor consumo para cada nivel de esfuerzo posible, lo que normalmente les hará equilibrar sus decisiones de asignación del tiempo entre trabajo y ocio en un menor nivel de trabajo y entre esfuerzo y pereza en un mayor nivel de pereza" (p. 197).

En el caso de los sectores más pobres, así prosigue esta lógica, puede ser eficiente un sistema de créditos, aunque este sistema tiene una serie de inconvenientes prácticos, por ejemplo, el costo de cobrar los créditos a mucha gente. Por estas razones, entonces, la solución que se impone es la de las subvenciones a la demanda de los consumidores 
de educación, para los estudios primarios y secundarios, y los créditos para la educación superior donde es más factible el cobro.

Como se ve en este esquemas, la política educativa, es decir, los aspectos normativos de la construcción de un sistema de educación, se deriva de cuestiones fácticas, ¿cuáles son los incentivos correctos para que los individuos trabajen más y se esfuercen más para maximizar utilidades? Como lo sabemos, en la filosofía moral y política contemporánea abundan las críticas al utilitarismo y la eficiencia como motivación central de las políticas públicas, las que van desde Bernard Williams a John Rawls, y Amy Gutman, por una parte, y por otra, desde Charles Taylor y Michael Sandel a Philip Pettit y los filósofos que se ligan al republicanismo. Nada hay, pues, más controvertible actualmente que la justificación política o moral del utilitarismo y la eficiencia, que aquí simplemente se asumen como la norma de lo que debe hacerse en educación. Los argumentos de estos filósofos críticos del utilitarismo subrayan, por una parte, que el utilitarismo no tiene éxito en medir todas las valoraciones humanas y en reducirlas a la utilidad y, por otra, que no califica como teoría moral, porque es incapaz de fundar un valor como la justicia, que debiera tener prioridad sobre las preferencias más o menos placenteras.

Por su parte, Charles Taylor subraya, en un influyente ensayo, que el utilitarismo no reconoce la diversidad de los bienes y en especial la distinción, inspirada en Harry Frankfurt, entre valoraciones fuertes, como las que subyacen a mi identidad personal, y las evaluaciones débiles, como las que tienen que ver con los deseos que me producen más placer o menos.

En realidad, en el primer texto recién citado nos encontrábamos también con algo diferente: con una condena directamente moral del ocio, que se contrasta con el trabajo y el esfuerzo como valores per se, con independencia de las formas de vida, las culturas y la historia. En esta perspectiva, entonces, la condena de la intervención del Estado es doble, no solo por el tipo de incentivos que promueve, sino también por su contribución a la gestación de una sociedad que genera propensión al ocio y, en general, a comportamientos no-económicos y anti-utilitarios: en una sociedad de mercado, lo moralmente correcto es esforzarse por pagar los servicios de alta utilidad privada ${ }^{10}$.

10 Este enjuiciamiento moral de los comportamientos anti-utilitarios es, obviamente simplista y unilateral, ya que los comportamientos sociales no guiados por la utilidad son una parte fundamental de la vida social, e incluso de la vida económica, como lo muestran los estudios clásicos de los autores institucionalistas como Thorstein Veblen y de antropólogos como Marcel Mauss, en su "Ensayo sobre el don". Más cerca nuestro están las críticas de la economía de mercado por autores como Karl Polanyi, que han contribuido a esclarecer la violencia y la desprotección social que se necesitan para convertir al trabajo en mercancía $\mathrm{y}$, por lo tanto, para lograr que los individuos se muevan solo por incentivos económicos. Ya en los Grundrisse de Marx en el año 1857 había una visión similar, en el célebre capítulo sobre las "Formas que anteceden a la producción capitalista". Roger Frydman, en su libro colectivo sobre L'economie-fiction y en sus ensayos en la revista MAUSS (Mouvement anti-utilitariste dans les sciences sociales) y Alain Caillé en su obra Critique de la raison 
Como decíamos más arriba, la defensa del modelo de mercado en educación también puede hacerse en términos del valor de la libertad. Es lo que hace, por ejemplo, Hernán Büchi otro pro-hombre chileno del neoliberalismo, en un ensayo titulado $L a$ transformación económica de Chile (Norma, 1993) y discutido recientemente por Juan Eduardo García Huidobro, Rocío Ferrada y Marcela Gil en un excelente ensayo titulado "La educación en la reflexión sociopolítica del período de la restauración de la democracia chilena (1988-2008)", del año 2010).

En este ensayo, Büchi celebra los éxitos de lo realizado por el gobierno militar en educación, también en términos de un cambio de mentalidad, lo que puede percibirse, según él, por ejemplo, en la pérdida de protagonismo de las universidades y del papel de conciencia crítica que se les atribuía. Esto tiene que ver, para Büchi, con las políticas de focalización que se sintetizan en que "El desafío en el área, fue dirigir los recursos a los sectores más necesitados y claramente ellos no estaban en la educación superior" (135). Otro criterio positivo del cambio del modelo se basó en considerar a los jóvenes que toman decisiones sobre sus estudios como seres libres y racionales: "Las personas son perfectamente capaces de discernir qué les conviene y qué no. Para eso no necesitan tutores como se cree al interior de los esquemas dirigistas. Tampoco se necesitan planificadores que proyecten y vaticinen la cantidad exacta de técnicos o profesionales que el país va a necesitar en diez o veinte años... Si alguien no obstante conocer la sobreoferta de profesionales de determinada especialidad insiste en matricularse en esa carrera y en asumir los costos de sus estudios, está en su pleno derecho" (136).

Por último, es interesante registrar, como lo hace García Huidobro, la crítica de Büchi a la inclusión del tema de los derechos humanos en el curriculum escolar, basada en la inexistencia de un consenso social sobre determinados valores; en ese caso, sostiene Büchi, el Estado no puede impulsar estos valores sin un riesgo: el de "manipular el perfil y los valores del cuerpo social, convirtiéndose en un ejercicio más del constructivismo social" (137).

En la mirada de Büchi, como puede percibirse claramente por estos extractos de su texto, es más bien el valor de la libertad definida con tonalidades libertarias hayekianas la que reemplaza al utilitarismo anterior.

Es interesante recalcar aquí dos puntos. El primero es la negativa percepción que dejan ver estos enunciados sobre el papel crítico de las universidades y, en general, podríamos decir, de los saberes críticos en educación, un papel que ha sido recientemente destacado y defendido con mucha fuerza por filósofas como Martha Nussbaum y Amy Guttman, que ven precisamente en el desarrollo de estos saberes críticos una garantía de que las instituciones de educación superior no solo reproduzcan y se adapten a los valores sociales predominantes en una sociedad de mercado, sino que tengan en cuenta posibilidades alternativas de organización social.

utilitaire (Paris, La Découverte: 1998) han desarrollado una crítica muy completa de la filosofía económica y social utilitaria en la economía y las ciencias sociales basada precisamente en las ideas de Marcel Mauss y Karl Polanyi, entre otros autores. 
El segundo punto es el papel conservador de los consensos, invocados por Büchi para contrarrestar la enseñanza de los derechos humanos en un país tan afectado por su violación sistemática como Chile, en la época dictatorial.

En realidad, estas defensas libertarias o utilitaristas del modelo educacional no reflejan todos los objetivos que se busca realizar con este tipo de políticas. A mi juicio, estas políticas constituyen también intentos de disciplinamiento social muy poderosos que se expresan, sobre todo, en el propósito explícito de despolitizar las instituciones de educación. Este fue, en realidad, el objetivo principal de la primera fase de la implementación de medidas autoritarias de la dictadura en el sistema educativo, pero continúan de otro modo con el esquema neo-liberal de mercado.

Expresiones de estas orientaciones se puede encontrar, por ejemplo, en el libro del Ministro de Educación de Pinochet, Alfredo Prieto, titulado La Modernización Educacional, de 1983, en donde el autor sostiene que con el papel central de las familias en la educación y con la idea de libertad de enseñanza que se deriva de esto como principio, puede evitarse también el escollo que significaría el rebrote de la actividad política y de la politización en el sistema público estatal. Si esto ocurre, asevera Prieto, "lo más probable es que va a surgir de inmediato la alternativa de un establecimiento que no esté afectado por tal problema, al cual los padres podrán llevar a sus hijos"11.

Se considera a veces como un logro histórico de la educación chilena la convivencia que hizo posible entre la educación estatal pública, con su raíz republicana y la educación privada, fundada en su origen en principios liberales esgrimidos en la defensa de la educación católica y luego de otras confesiones y proyectos.

El problema es que es mucho más difícil, me parece, conciliar el actual modelo de educación centrada en el mercado, con una educación pública estatal de calidad.

Aquí de nuevo vuelvo al texto de Gerardo Jofré que cité más arriba, pero esto es solo un ejemplo, uno de los más extremos, tal vez.

Según Jofré, el modelo de la dictadura, basado en la libertad de elección de las familias, las subvenciones a la demanda y los créditos para los estudiantes universitarios, plantea, además, una importante dificultad para el sistema: ¿cómo determinar qué

11 Podemos encontrar ideas similares en una columna de Alvaro Bardón en El Mercurio del 10 de julio de 1980. En esta columna, Bardón, también ministro de Pinochet, sostiene que la solución del problema del activismo estudiantil pasa por el cobro de los estudios universitarios. A la luz de los movimientos estudiantiles recientes contra el cobro de la educación superior, liderados por estudiantes que pagan por la educación, los pronósticos y la lógica economicista del exministro resultaron falsos. 
estudiantes deben ser subsidiados y cuáles no? A Jofré le parece que la mejor solución de este problema es que los mismos postulantes al sistema se autoclasifiquen y no que sean asignados a uno u otro esquema por el Estado. Pero señala muy correctamente que las personas pueden autoclasificarse solo si perciben diferencias entre el sistema subsidiado, municipal o subvencionado, y el sistema privado pagado.

"Esto significa - nos dice- que para que exista autoclasificación, la cual no adolece de ninguno de los serios inconvenientes de la selección de beneficiarios por el Estado, debe admitirse que la educación subvencionada será de calidad inferior que la pagada (el destacado es mío, C. R.). Esta idea suena chocante, pero no es sino la realidad que existe en cualquier parte del mundo. Si no existiera esta diferencia, nadie asistiría a escuelas pagadas" (Letelier 1895, p. 213). Pero -agrega Jofré- “el problema es más complicado: en la medida en que suba la calidad de las escuelas subvencionadas, tenderá a vaciarse un contingente de alumnos desde las pagadas a aquellas. Si el Estado aumenta persistentemente las subvenciones, tiende a que toda la población termine educándose en forma gratuita. Esto puede parecer atractivo...pero significa echar una pesada carga sobre las espaldas de la economía, carga que terminará por empobrecer a la generalidad de los habitantes del país..." (Letelier 1895, p. 215).

La eufemística conclusión que extrae Jofré de este análisis, que a él mismo le parece algo exagerado, no es que la calidad de las escuelas subvencionadas deba caer, como se deriva en efecto lógicamente del argumento para la auto clasificación de los beneficiarios de los subsidios...Sólo debe "administrarse". Lo que no evita que en ciertas circunstancias pueda ser conveniente "contener el aumento de la calidad de la educación subvencionada para obtener a largo plazo una mayor calidad que la que se obtendría expandiéndola indiscriminadamente" (Letelier 1895, p. 214).

Por último -sostiene- es importante subrayar la inconveniencia de tener un sistema educacional demasiado polarizado: los subsidios, por una parte, y por otra la educación pagada. Es en base a estas consideraciones que Jofré propone mecanismos que hagan al sistema más flexible para que los padres que pueden pagar algo más que la subvención estatal a ciertos colegios, lo hagan. De esta manera es que fundamenta lo que se llama "financiamiento compartido".

Es claro que, en la perspectiva de Jofré, el sistema delineado -y que coincide a grandes rasgos con el implementado en Chile por Pinochet- es el menos malo y el que debe implementarse. Para él es evidente también que sus lineamientos generales "los satisfacen mejor los establecimientos privados que los estatales, y entre aquellos, mejor los subvencionados que los propiamente estatales".

¿Cuál es el lugar y el papel de la educación pública estatal en este esquema? La primera respuesta que a uno le viene a la mente es que prácticamente ninguno, salvo hacer posible la educación de los sectores más vulnerables de la población, a condición de que sea rentable que se eduquen. Pero como acabamos de leerlo en el texto de Jofré, este tipo de educación no puede ser de buena calidad. Ahora bien, si esto es así, entonces este diseño no apunta verdaderamente a un sistema mixto -como el sistema que existió en Chile hasta 1973-, sino a un sistema privado que compensa -sin gran calidad-a los sectores más pobres, que no satisfacen los criterios de la racionalidad económica. 
Y en verdad es esta la respuesta que se deduce del pensamiento de los autores del modelo del mercado, cuya estructura -a través de normas jurídicas de nivel constitucional como la LOCE y la LGE- continúa hasta hoy, con pocos cambios, algunos de los cuales lo profundizan aún más, como el financiamiento compartido en la educación general y el crédito con aval del Estado en la educación universitaria.

Un breve texto final -aunque anterior a los que recién citamos-puede servirnos para mostrar esto que acabamos de decir.

El texto en cuestión lo extraemos de un editorial de la revista Realidad, publicado en 1982 y titulado "Institucionalidad universitaria: avances sustantivos entre contradicciones"12. Comentando el mecanismo de financiamiento fiscal indirecto a los 20.000 mejores puntajes de la prueba de selección para el ingreso a la universidad, incluido en los Decretos con Fuerza de Ley de Pinochet el año 1981, se dice que: "El aludido financiamiento estatal indirecto aparecía como un ingeniosos medio para favorecer las ...iniciativas particulares, contribuyendo a la gradual desestatización de nuestra estructura universitaria" (p. 2) (La cursiva es mía). Para la revista, sin embargo, este propósito ha quedado frustrado por el momento por uno de los últimos DFL, que impide que este beneficio pueda ser aprovechado por las universidades privadas de reciente creación. Después de una defensa sin matices de estas universidades, sostiene el artículo que los argumentos expuestos por la revista, "mueven a esperar que este retroceso sea remontado lo más pronto posible, restableciendo en su esencia la norma original pertinente que, tanto en doctrina como en la práctica, es la más congruente con la nueva institucionalidad universitaria impulsada por el actual Gobierno (p. 3).

Parece claro que un texto como este reafirma, entre líneas, lo que venimos diciendo: el objetivo del modelo de mercado en educación en Chile ni es ni fue hacer posible un sistema mixto estatal y privado, sino conducir a "una gradual desestatización" del sistema universitario y del sistema educacional en general.

Las recientes medidas en educación superior reafirman este propósito, por lo menos para el sector, porque se puede contribuir de muchas maneras a "desestatizar la educación superior". Una de esas maneras es disminuir la oferta de educación superior pública estatal, eliminando los que se han llamado "aportes basales". Otra manera es disminuir el valor de los créditos y aumentar las becas estudiantiles, para "todas" las instituciones, entregando entonces recursos del Estado a las instituciones privadas. Se aumenta así el gasto estatal, pero se beneficia a las instituciones privadas que se suponía, en la teoría económica, que iban a hacer posible una mayor racionalidad económica en los actores y a aliviar el gasto educacional, dejando libres recursos para otras legítimas demandas insatisfechas de la población.

12 La revista Realidad, fundada a comienzos de la década de los 1980, es un tribuna de pensamiento dedicada a la difusión de las ideas neo-liberales. Escribían habitualmente en ella dirigentes políticos e intelectuales de la derecha como Jaime Guzmán, José Piñera, Hermógenes Pérez de Arce, Pablo Barahona y Ernesto Illanes, entre otros. 


\section{Referencias bibliográficas}

Friedman, Milton y Rose (1980), Libertad de elegir. Hacia un nuevo liberalismo económico. México: Grijalbo.

Letelier, Valentín (1895), "Los pobres", en el diario La Ley, Santiago, $1^{\circ}$ de enero de 1896.

Nicolet, Claude (1982), L'Idée republicaine en France. Paris: Gallimard.

Prieto, Alfredo (1983), La modernización educacional. Santiago: Ediciones de la Universidad Católica, p. 94.

Ruiz, Carlos (2010), De la República al mercado. Ideas educacionales y política en Chile. Santiago: LOM Ediciones.

Skinner, Quentin(1997), “The state”, en RobertE. Goodin y Philip Pettit, Contemporary political philosophy. Ananthology. Oxford y Malden, Massachusetts: Blackwell Publishers.

Spitz, Jean-Fabien (2005), Le moment republicain en France. Paris: Gallimard.

(2008), “El republicanismo francés”. En C. Ruiz y M. García de la Huerta, comp., República, liberalismo y democracia. Santiago: LOM Ediciones, 2011, p. 140.

Wahnich, Sophie (2011), "Defender la gratuidad escolar hoy (como ayer)", en C. Gutiérrez, A. Martin, C. Ruiz y P. Vermeren, Pasado y Presente de la Educación Pública. Miradas desde Chile y Francia. Santiago: Catalonia. 